Cite this: Phys. Chem. Chem. Phys., 2011, 13, 1754-1761

www.rsc.org/pccp

PAPER

\title{
Freezing single molecule dynamics on interfaces and in polymers
}

\author{
Stefan Krause, ${ }^{a}$ Pedro F. Aramendia, ${ }^{b}$ Daniela Täuber ${ }^{a}$ and \\ Christian von Borczyskowski ${ }^{a}$
}

\author{
Received 4th September 2010, Accepted 3rd November 2010 \\ DOI: $10.1039 / \mathrm{cocp01713b}$
}

Heterogeneous line broadening and spectral diffusion of the fluorescence emission spectra of perylene diimide molecules have been investigated by means of time dependent single molecule spectroscopy. The influence of temperature and environment has been studied and reveals strong correlation to spectral diffusion processes. We followed the freezing of the molecular mobility of quasi free molecules on the surface upon temperature lowering and by embedding into a poly(methyl methacrylate) (PMMA) polymer. Thereby changes of optical transition energies as a result of both intramolecular changes of conformation and external induced dynamics by the surrounding polymer matrix could be observed. Simulations of spectral fluctuations within a two-level system (TLS) model showed good agreement with the experimental findings.

\section{Introduction}

Single molecule spectroscopy (SMS) using probe molecules in soft matter $^{1}$ and at interfaces ${ }^{2,3}$ has been extensively used to study molecular dynamics. ${ }^{4}$ In material and biological sciences single emitters which are extremely sensitive to interactions with the environment and related dynamics constitute ideal nano-probes for polymers, ${ }^{5,6}$ amorphous solids, ${ }^{7}$ glasses ${ }^{8-11}$ and proteins. ${ }^{12,13}$ Heterogeneous media result often in fluctuations of spectroscopic observables such as emission, absorption, luminescence decay rates, translational or rotational motion. Heterogeneities are either of static or dynamic nature. With ensemble experiments it is in most cases impossible to discriminate between these two assignments. SMS, however, is an ideal tool to circumvent such limitations.

Recent SMS investigations of heterogeneous media can be either related to low temperature experiments with high spectral resolution, ${ }^{8-10,14,15}$ determining spectral diffusion and spectral line width, or to experiments at room temperature, which in most cases deal with rotational diffusion ${ }^{16-18}$ or fluorescence lifetime fluctuations. ${ }^{19-22}$ Investigations of spectral diffusion and the related time dependency of optical transition energies are rare at elevated temperatures since intrinsic line broadening precludes from investigating relatively small spectral jumps which are typical for interactions of a chromophore with the environment. However, molecules with pronounced internal degrees of freedom such as intra-molecular charge transfer or internal deformations evidence spectral fluctuations detectable even at room temperature and give the opportunity to follow dynamical processes of the nanoscopic

\footnotetext{
${ }^{a}$ Institute of Physics and nanoMA (Center for nanostructured Materials and Analysis), Chemnitz University of Technology, 09107, Chemnitz,Germany.E-mail: stefan.krause@physik.tu-chemnitz.de; Fax: + 49 (0)371 531 837418; Tel: + 49 (0)37153137418

${ }^{b}$ Dept. Química Inorgánica, Universidad de Buenos Aires, C1428EHA, Buenos Aires, Argentina
}

environment by time dependent measurement of the fluorescence emission spectra of the single emitters. ${ }^{23-25}$

The intentions of the present paper are twofold. On one side, we aim at first steps to unravel how internal molecular degrees of freedom will couple as a function of temperature to external properties of the amorphous environment which can be twolevel systems, ${ }^{8}$ free volumes, ${ }^{22}$ meta basins ${ }^{20}$ or low frequency vibrations. $^{7}$ Secondly, we will investigate the behaviour of a probe molecule when changing the constraints imposed by the environment for example by replacing a heterogeneous interface such as $\mathrm{SiO}_{2}$ by a polymer matrix.

A suitable molecular system for our goals should have well defined conformers, which is e.g. realized for perylene diimide (PDI) molecules with appropriate substituents. ${ }^{22,26,27}$ First single molecule experiments on this type of dyes revealed strong spectral fluctuations (spectral diffusion) due to the mobility of attached phenoxy (bay) groups capable to change their positions relative to the chromophoric perylene backbone and the properties of the backbone itself. ${ }^{28-30}$ As a result the molecules exhibit differences in the optical transition energy of up to $0.27 \mathrm{eV}$ making spectral fluctuations easily observable also under ambient conditions.

Though a qualitative assignment of the observed spectral fluctuations to specific dynamic interactions is in most cases so far reported quite obvious, a quantitative analysis is often missing. Basically such a quantitative analysis will only be successful via correlation of several spectroscopic observables such as wavelength and fluorescence intensity including temperature variation. Recently we have shown that such correlations exist. ${ }^{30}$ This enables us to investigate in the present paper quantitatively the freezing of spectral dynamics both upon lowering temperature and/or upon transferring the probe molecules from an interface allowing for a high degree of mobility into a polymer which restricts on the one hand mobility to a larger extent but enhances small fluctuations due to polymer dynamics on the other. 


\section{Experimental}

Dialkyl (DAP), monopyridyl (PP) and dipyridyl perylene diimides (DPP) were synthesized by Würthner and Sautter. ${ }^{27}$ (For structures see Fig. 6.) To prepare single molecule experiments, glassware was cleaned with acetone and ethanol followed by heating up to $60{ }^{\circ} \mathrm{C}$ in a Hellmanex II solution. Small amounts of the original solution of dye molecules in toluene were diluted in spectroscopic toluene (Merck) to achieve a concentration of about $10^{-11} \mathrm{~mol}^{-1}$. To ensure clean substrates, silicon wafers with a $100 \mathrm{~nm}$ thick thermally grown oxide layer (ENAS, Chemnitz) were first treated with acetone and ethanol and then placed for at least one hour into a mixture of $3: 2 \mathrm{H}_{2} \mathrm{SO}_{4}$ and $\mathrm{H}_{2} \mathrm{O}_{2}$ in an $80{ }^{\circ} \mathrm{C}$ ultrasonic bath. Remaining contaminations on the substrates were eliminated by annealing those 20 to $30 \mathrm{~s}$ with a gas flame. The purity with respect to luminescence was verified with a standard wide field microscope (excitation wavelength $\lambda_{\mathrm{ex}}=514 \mathrm{~nm}$ ). Spatially well separated single molecules on the substrate were achieved by spin coating the toluene solution at $3000 \mathrm{rpm}$ followed by evaporation of the remaining toluene. The preparation of PMMA samples followed the same procedure. To achieve a $5 \mathrm{~nm}$ to $10 \mathrm{~nm}$ thick polymer film on the sample, $20 \mathrm{mg}$ of PMMA were dissolved in $4 \mathrm{ml}$ of the final mixture of toluene and dye molecules. Spin coating was performed under same conditions as for pure $\mathrm{SiO}_{2}$ samples. Vacuum pumping for at least $1 \mathrm{~h}$ in the cryostat at room temperature ensured complete toluene evaporation.

Single molecule spectroscopy and microscopy experiments were performed with a home built confocal laser scanning microscope. A $532 \mathrm{~nm} \mathrm{Nd:YAG} \mathrm{laser} \mathrm{(Spectra} \mathrm{Physics)} \mathrm{was}$ used as the excitation light source. The beam was scanned with the scanning mirror of a control scan stage (Newport) and then focused through the cryostat cover glass $(0.5 \mathrm{~mm})$ onto the sample with a $63 \times$ cover glass corrected objective (Carl Zeiss Jena) with a numerical aperture of NA $=0.75$. All experiments were performed under vacuum condition $\left(10^{-5} \mathrm{mbar}-10^{-6} \mathrm{mbar}\right)$ in a Janis Research Cryostat. For low temperature measurements the Cryostat was cooled with liquid helium to $4.2 \mathrm{~K}$. The fluorescence signal was separated from the excitation light via a $542 \mathrm{~nm}$ long pass filter (Omega Optical). A 30 : 70 beam splitter allowed for recording simultaneously images with an avalanche photodiode (Perkin Elmer) and single molecule spectra with a monochromator (300 lines per $\mathrm{mm}$ grating, Acton Research Corporation, SpectraPro 275) equipped with a liquid nitrogen cooled CCD camera (Princeton Instruments, ST-121) allowing for spectral resolution of $(2.3 \pm 0.2) \mathrm{nm}$ at $600 \mathrm{~nm}$ wavelength and for a $200 \mu \mathrm{m}$ entrance slit (note that the positioning accuracy of spectral emission peaks is slightly improved for high signal to noise ratio). Evaluation of single molecule spectra was performed by an adapted Matlab fitting algorithm.

\section{Results}

PP has in comparison to DPP only one pyridyl group. The other one is replaced by a single butyl chain. It is expected that - as shown in Fig. 1-PP and DPP are close to an upright position of the transition dipole moment as might be confirmed by recent experiments according to the procedure suggested by Hohlbein and Hübner. ${ }^{31}$ We already investigated the orientation of these types of molecules by means of such 3D
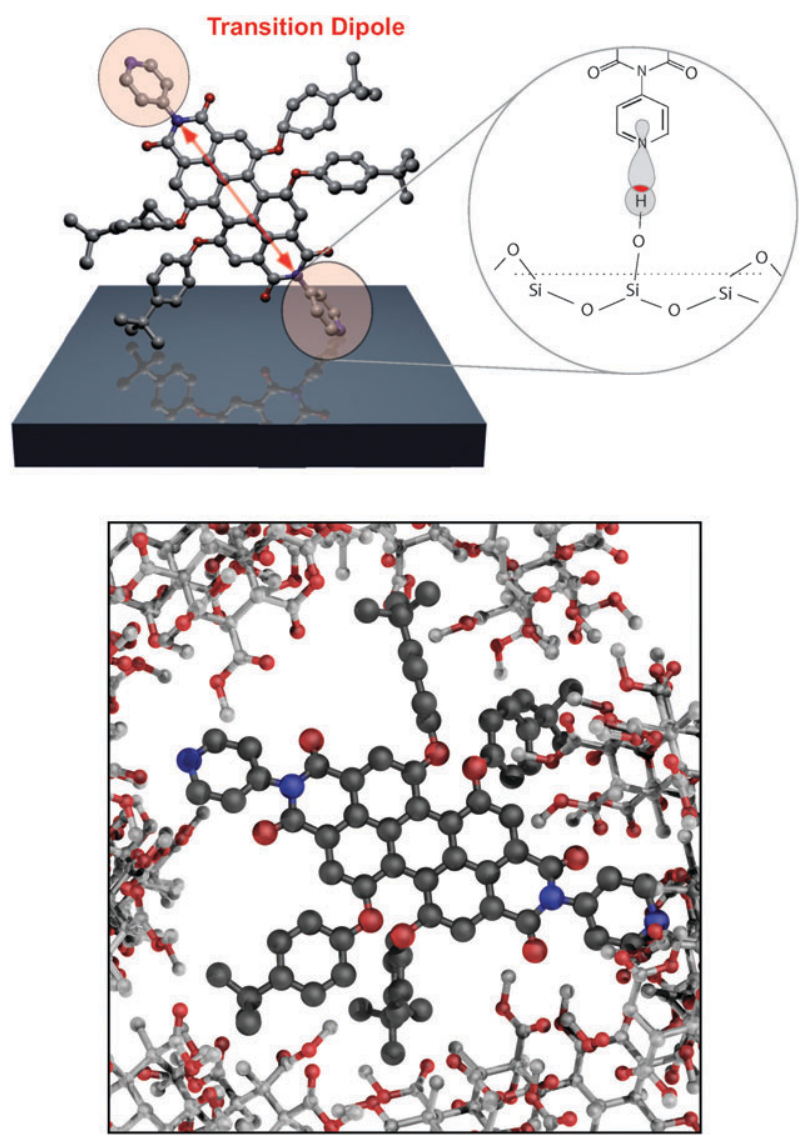

Fig. 1 Top: Structure of $N, N^{\prime}$-bis(pyridyl)-1,6,7,12-tetrakis(3-hydroxyphenoxy)perylene-3,4:9,10-tetra-carboxylicaciddiimide (DPP). The figure shows an extended conformation of DPP. The pyridyl groups (see coloured circles) allow for directed attachment of the molecule to the silanol groups on the $\mathrm{SiO}_{2}$ surface (see enlarged scale). Scheme of a PMMA embedded DPP molecule. Bottom: The phenoxy groups in the bay position allow for a strong coupling to the polymer environment.

orientation measurements. ${ }^{32}$ The experiments revealed indeed a side-on orientation for the molecules functionalized with pyridyl groups such as PP and DPP. As these measurements yielded a large amount of interesting data it would go beyond the scope of this paper. Presently detailed experiments related to this subject are underway.

In the case of DAP even both groups are replaced by butyl chains. The structural formula of DPP is depicted in Fig. 1. DPP, PP and DAP (structural formulas are additionally shown in Fig. 6) contain 4 bay groups which give rise to at least 5 conformers. ${ }^{29}$ They are different regarding their relative orientation with respect to the perylene moiety. The two limiting conformers are those where all bay groups are extended or folded, with respect to the perylene backbone. The intermediate ones are those where only 1,2 or 3 bay groups are folded. ${ }^{29}$ While changes in conformation on $\mathrm{SiO}_{2}$ due to transitions between different conformers have been observed recently, the situation is expected to be different for a polymer host which strongly restricts large movements of the phenoxy groups. The question whether a coupling of the polymer dynamics to the optical transition dipole is possible or even useful for characterisation will be further discussed. 


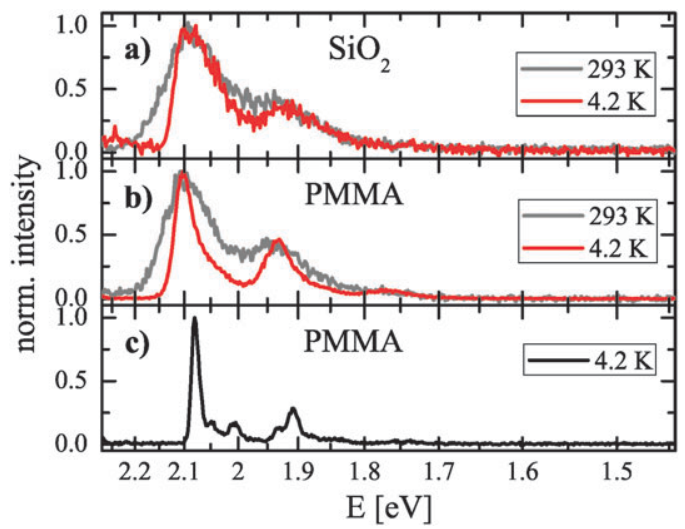

Fig. 2 Fluorescence emission spectra of single DPP molecules at 293 and $4.2 \mathrm{~K}$ on $\mathrm{SiO}_{2}$ (a) and in PMMA (b and c), respectively. Excitation wavelength $\lambda_{\mathrm{ex}}=532 \mathrm{~nm}$ and laser power $P_{\mathrm{ex}}=20 \mu \mathrm{W}$. The example in (c) shows a fluorescence emission spectrum which can be observed for only $5 \%$ of the investigated molecules in PMMA at $4.2 \mathrm{~K}$.

We investigate single PDI molecules deposited on to a $\mathrm{SiO}_{2}$ surface or embedded into thin films of PMMA. Fig. 2 shows typical fluorescence emission spectra of single DPP molecules at $293 \mathrm{~K}$ and $4.2 \mathrm{~K}$ on a $\mathrm{SiO}_{2}$ surface (top) and in PMMA (middle), respectively. Spectral shape and line width are only slightly reduced upon temperature decrease. This is very similar to recently reported results. ${ }^{33}$ Such behaviour is observed for most of the molecules which cover in total a transition energy region of $1.94 \mathrm{eV}$ to $2.21 \mathrm{eV}$. $^{32}$ The broad range of emission is due to conformational changes of the bay groups with respect to the chromophoric PDI backbone. ${ }^{29,30}$ In PMMA at $4.2 \mathrm{~K}$, a few molecules show narrow spectra which are within the range of our experimental resolution as shown for comparison in the bottom graph in Fig. 2. At $293 \mathrm{~K}$, and especially on $\mathrm{SiO}_{2}$, such narrow spectra could not be observed.

This broadening is due to spectral diffusion even at $4.2 \mathrm{~K}$ as is shown in Fig. 3(a) for spectrally resolved wavelength jumps of PP in PMMA. Spectral fluctuations could be detected because they occurred on time scales larger than the integration time for a single spectrum. The fluorescence time trace at the top of Fig. 3 shows fluctuations both in spectral positions and fluorescence intensities. Spectra with an integration time of $0.5 \mathrm{~s}$ are shown at various observation times in Fig. 3(b), while a "sum"-spectrum of the 4 spectra is shown below. The individual spectra are still considerably broadened. The time trace reveals that at least two types of spectral fluctuations are evident: large spectral jumps of $60 \mathrm{meV}$, which occur rarely, and frequent jumps of about $15 \mathrm{meV}$ and less. The intensity fluctuations are correlated with the spectral jumps. Owing to such spectral fluctuations, unbroadened line widths cannot be detected as long as the integration time is larger than the time scale of spectral jumps. The sum spectra as an example represents such a resulting line broadening.

To investigate the time dependent spectral processes more in detail we fitted the fluorescence spectra of single molecules by up to four Gaussian lines yielding the peak positions, line widths and amplitudes. Fig. 4 shows the distribution of line widths (FWHM) of the highest energy emission band for the two environments at $4.2 \mathrm{~K}$ and $293 \mathrm{~K}$.

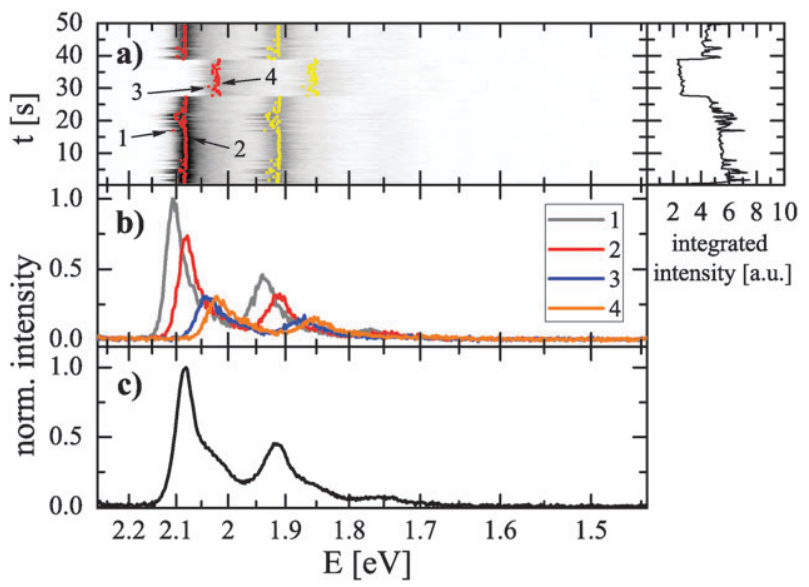

Fig. 3 (a) Spectral time trace of PP in PMMA at 4.2 K. Red (yellow) dots mark the positions of the respective fluorescence origin (vibronic side band). Consecutive spectra are plotted in a gray scale map and exhibit small fluctuations of about $15 \mathrm{meV}$ during the whole observation time and two large spectral jumps of about $60 \mathrm{meV}$. The numbers 1-4 mark single fluorescence spectra which are representative for the four observed spectral states. They are displayed in (b). The time trace of the integrated intensity shows clear correlation of spectral jumps and intensity changes. (c) Sum spectra of all single frames of (a).

In principle comparison of ensemble FWHMs and FWHMs of a single emitter observed for a very long time allows us to investigate the break down of ergodicity but it requires very long measuring times for one single molecule. A single molecule will especially for long times explore the same energy landscape like the ensemble as long as it is not restricted by its nanoscopic environment to a certain energy range (molecule 1 in Fig. 4). The environment might thus prevent single molecules from exploring the total energy landscape (molecule 2 in Fig. 4). In that case ergodicity breaks down. From the observations we conclude that for some of the molecules ergodicity applies for others it does not.

The overall behaviour shown in Fig. 4 seems to be quite complex. At $T=293 \mathrm{~K}$ both the covered range and the width of the related line width distribution are larger for $\mathrm{SiO}_{2}$ (especially towards large FWHMs) as compared to those for PMMA. From this we conclude that the extent of spectral diffusion is, on the scale of our observation time, larger for $\mathrm{SiO}_{2}$ than for PMMA. This is reasonable, since PMMA will reduce the degree of spatial (and rotational) mobility for DPP which is apparently more "floppy" on a $\mathrm{SiO}_{2}$ surface. The extremely broad line widths on $\mathrm{SiO}_{2}$ are due to the fact that on the scale of the integration time of $0.5 \mathrm{~s}$ DPP explores a broader range of conformers. This is in line with observations of Ishikawa and coworkers from lifetime fluctuations and intensity time traces. ${ }^{34}$

The behaviour seems to become quite opposite at $4.2 \mathrm{~K}$. While on $\mathrm{SiO}_{2}$ the average FWHM is reduced by at most a factor of 2 while maintaining a similar width in distribution (excluding the tail at large line widths for $293 \mathrm{~K}$ ), the average width is less reduced in PMMA, but the width of the distribution is apparently much broader at $4.2 \mathrm{~K}$ as compared to $293 \mathrm{~K}$. The related distributions are close to Gaussian ones in all cases besides the one for DPP in PMMA at $4.2 \mathrm{~K}$, which seems to be a superposition of several distributions. This interpretation implies that PMMA freezes 

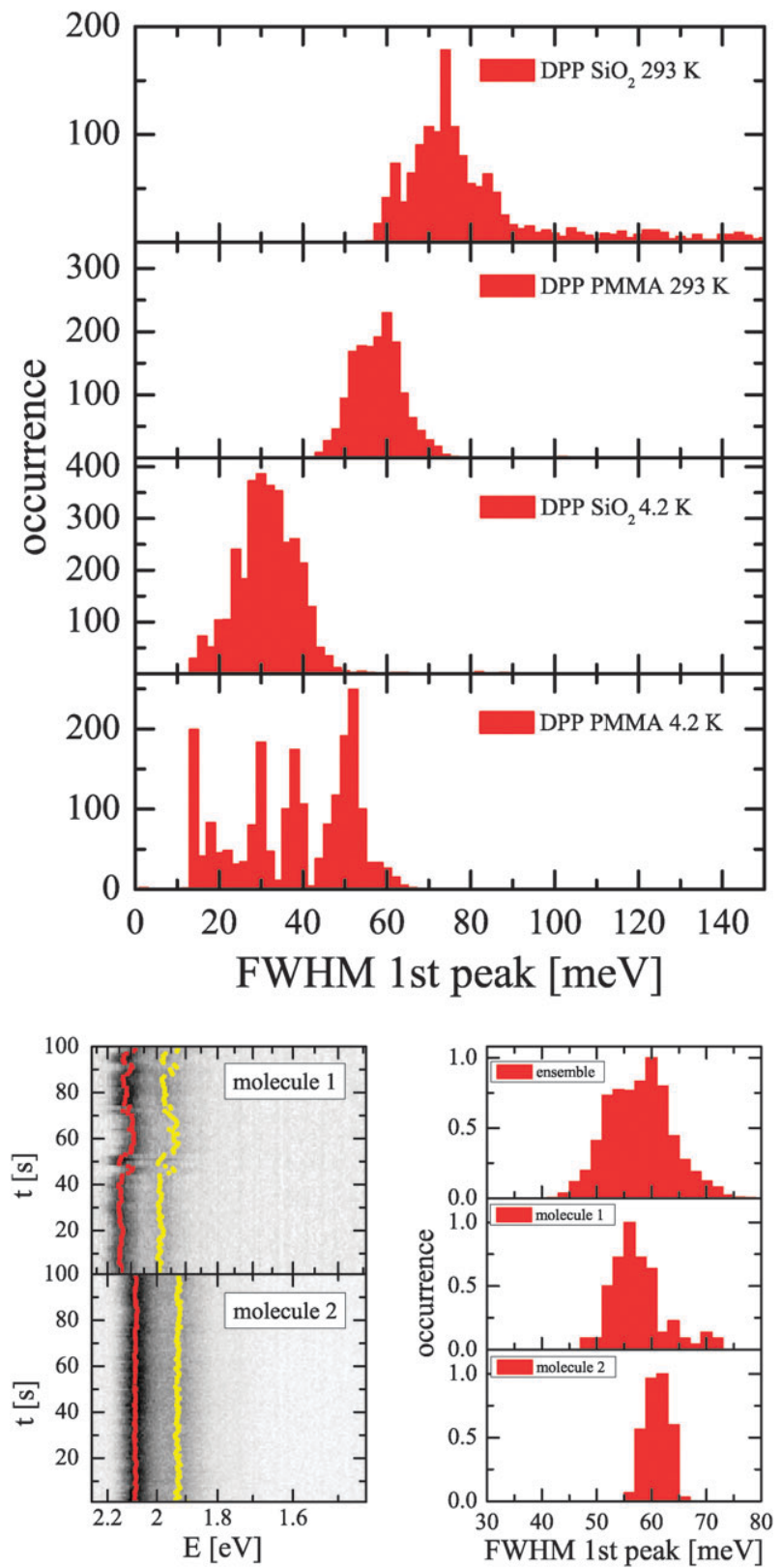

Fig. 4 Top: full width at half maximum (FWHM) distributions for DPP fluorescence emission spectra on $\mathrm{SiO}_{2}$, in PMMA, for $293 \mathrm{~K}$ and $4.2 \mathrm{~K}$. Spectra were collected in time traces for $25-50$ molecules. For each molecule a maximum of 200 frames were recorded or less for bleaching molecules. Bottom: two time dependent single molecule spectra of DPP in PMMA at $293 \mathrm{~K}$. The spectrum of molecule 1 shows strong spectral fluctuations where molecule 2 emits at constant wavelength during the observation time. As a result molecule 1 represents the ensemble distribution of FWHMs for the first emission peak more precisely (see distributions for DPP in PMMA at $293 \mathrm{~K}$ on the right).

at least a few DPP-environment configurations with FWHM differences of a factor of 5. The temperature behaviour of FWHM distribution in PMMA can be explained by a coalescence of different conformations due to temperature increase.

The overall behaviour of FWHM in connection with the rare but nevertheless present narrow line spectra suggests that spectral diffusion, on a time scale faster than $0.5 \mathrm{~s}$, covers a range of about $30 \mathrm{meV}-60 \mathrm{meV}$, nearly independent of temperature. In PMMA some of the DPP molecules maintain at $4.2 \mathrm{~K}$ the same FWHM as at $293 \mathrm{~K}$ while others exhibit line widths as narrow as the experimental resolution (see the bottom spectrum in Fig. 2).

Spectral diffusion slower than $0.5 \mathrm{~s}$ can be detected as discrete spectral jumps (see Fig. 3). To analyze these jumps, we use the difference of the maxima of two consecutive emission spectra $\Delta E=E_{i+1}-E_{i}$ where $i$ denotes the number of the spectrum within the spectral time trace. Fig. 5 shows the distribution of spectral jumps for $\mathrm{SiO}_{2}$ and PMMA at $293 \mathrm{~K}$ and $4.2 \mathrm{~K}$ on a $\log$-lin scale. The noise limited jump width is close to $\pm 3 \mathrm{meV}$ which is obtained for DPP in PMMA at $4.2 \mathrm{~K}$. Spectral jumps larger than $\pm 25 \mathrm{meV}$ are in all cases nearly completely frozen at $4.2 \mathrm{~K}$. For $\mathrm{SiO}_{2}$ at $293 \mathrm{~K}$ we observe spectral jumps of up to $120 \mathrm{meV}$ for DPP. Similar large changes in the optical transition energy have for example also been reported for conjugated polymers and were addressed to strongly distance dependent interactions of two or more chromophores $^{35,36}$ in a polymer chain. As we investigate well separated single molecules we assign conformational changes of bay groups of DPP as the reason for the observed fluctuations. ${ }^{29}$ Only very few large jumps between $25 \mathrm{meV}$ and $120 \mathrm{meV}$ are observed for DPP in PMMA (see Fig. 3 (top)).

The jump width distribution at $4.2 \mathrm{~K}$ is shown on an enlarged energy scale in Fig. 6 together with additional data for PP and DAP in PMMA. The distributions show in all cases a characteristic central shape, which can be fitted with three Gaussian distributions (one for the noise and a second and third for small spectral diffusion resulting in positive or negative energy differences left and right of the center) and in the case of PP and DAP two additional "side-bands" separated by $15 \mathrm{meV}-20 \mathrm{meV}$ from the central peak of the distribution. We like to mention that the range of spectral diffusion is in all cases larger for PP and especially for DAP than for DPP. We assign this to the replacement of the pyridyl group(s) by one or two flexible butyl chains, preventing a double anchoring of the molecules (DAP) to the matrix. It is obvious from Fig. 6 that

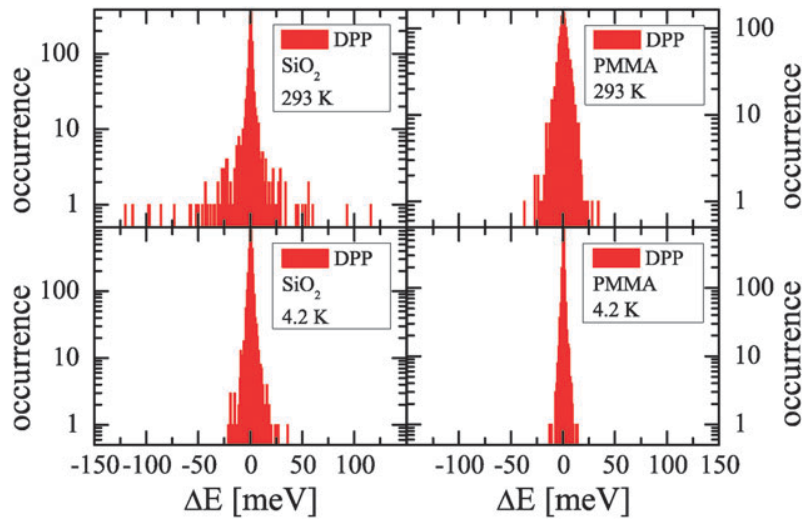

Fig. 5 Distribution of spectral jump widths for $\mathrm{DPP}$ on $\mathrm{SiO}_{2}$, and in PMMA, at $293 \mathrm{~K}$ and $4.2 \mathrm{~K}$. The distributions are shown on a log-in scale to emphasise large spectral jumps which occur with a very low probability. 


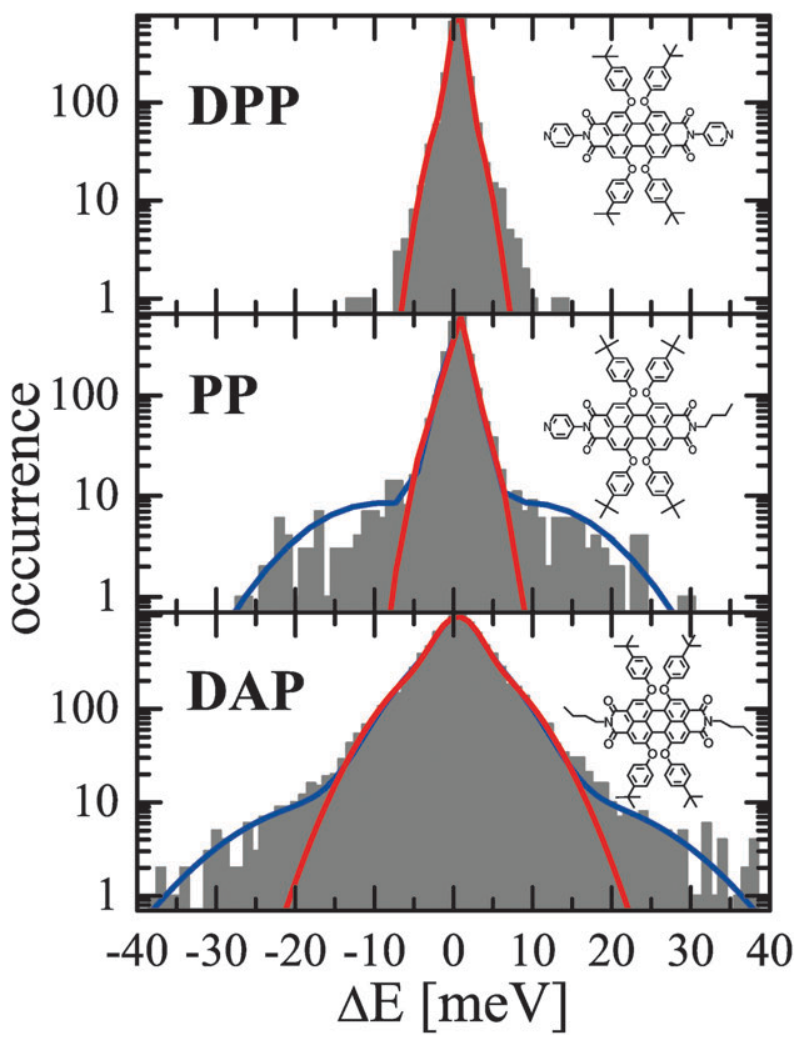

Fig. 6 Comparison of jump width distributions for DPP, PP and DAP molecules in PMMA at $4.2 \mathrm{~K}$. The center peak was fitted by 3 Gaussian functions and represents small spectral fluctuations and broadening through noise. In the case of PP and DAP two additional Gaussian functions represent spectral jumps between 3 $(-3) \mathrm{meV}$ and $40(-40) \mathrm{meV}$.

the butyl chain(s) in the case of PP and DAP are responsible for the coupling to external dynamics of the surrounding polymer since they enhance spectral diffusion processes strongly.

\section{Discussion}

To describe spectral diffusion more quantitatively we borrow ideas from translational diffusion analytics. The model of complementary cumulative distributions allows in the case of 2-dimensional spatial diffusion the determination of diffusion coefficients. ${ }^{37}$ As a matter of fact, the 2-dimensional spatial diffusion transfers to a 1-dimensional one in energy (wavelength) space. To emphasize the difference between spatial and spectral diffusion we label the spectral entities with the suffix "s". Formally we define in this way diffusion coefficients very similar to those in spatial diffusion. For the model of complementary cumulative distributions it is not necessary that the system is ergodic. The complementary cumulative distributions are derived from a straightforward statistical analysis. Of course diffusion coefficients will differ between the time average measurement and the ensemble average measurement if the system is not ergodic.

However, one has to be aware that e.g. fast diffusion does not imply that spectral jumps occur very frequently, but that large (rare) spectral jumps are effectively realised per time unit $\tau$.
In the case of spectral diffusion we are interested in the cumulative distribution of the squared amplitude of spectral jumps. This gives us the opportunity to gain exact spectral diffusion coefficients despite the fact that the obtained spectral trajectories consist at most of 200 data points for a single molecule which is not sufficient to extract values according to the model of mean squared displacement. ${ }^{38}$ To proceed we define a spectral diffusivity

$$
d_{\mathrm{s}}=\frac{\Delta E^{2}}{2 \tau}
$$

where $\Delta E^{2}$ is the square of the energy difference of two consecutive spectra belonging to one single molecule and $\tau$ represents the time difference between the two spectra. We calculate the spectral diffusivity between every pair of data points and sort them in descending order according to the probability of the respective occurrence. The complementary cumulative distribution

$$
C_{\mathrm{s}}\left(d_{\mathrm{s}}\right)=1-P_{\mathrm{s}}\left(d_{\mathrm{s}}\right)
$$

with $P_{\mathrm{s}}\left(d_{\mathrm{s}}\right)$ as the cumulative distribution representing the probability to measure a value smaller than $d_{s}$, can be obtained by plotting the ratio $j / N$ as a function of the diffusivity ( $j$ is the position of the $j$ th data point in the sorted diffusivity row and $N$ the overall number of events). Despite the fact of a finite energy space explored by the different molecule-environment conformations, a spectral diffusion coefficient $D_{\mathrm{s}}$ can be determined. In cases of fast diffusion processes the cumulative distribution curve would exhibit a small negative gradient and thus a high diffusion coefficient $D_{\mathrm{s}}$, whereas slow processes lead to a large negative gradient and a related small $D_{\mathrm{s}}$ as has been shown in the case of translational diffusion in liquids. ${ }^{37,39}$

Application of this formalism to the spectral diffusion of DPP results in complementary cumulative distributions (complementary functions) as shown in Fig. 7 at $293 \mathrm{~K}$ and
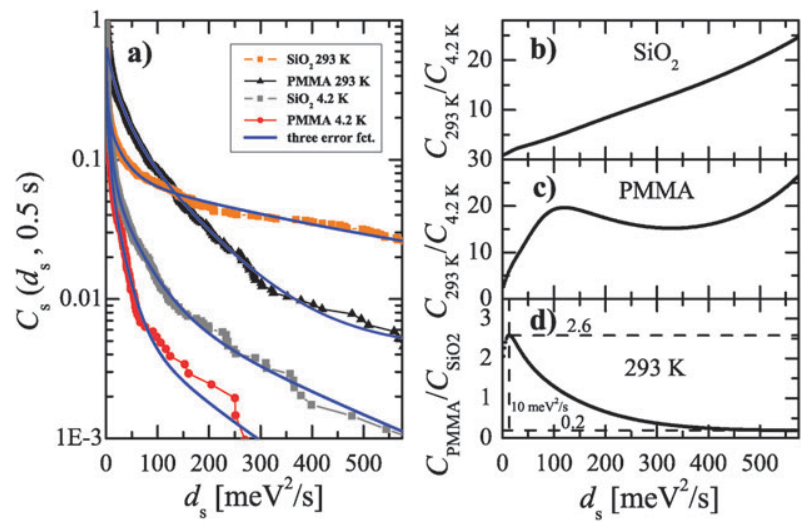

Fig. 7 (a) Complementary functions for spectral jump widths of DPP molecules on $\mathrm{SiO}_{2}$, in PMMA, for $293 \mathrm{~K}$ and $4.2 \mathrm{~K}$. The blue lines represent the fits to the sum of three error functions. (b-d) Ratio of the fitting functions for $\mathrm{SiO}_{2}(293 \mathrm{~K}$ and 4.2 K), PMMA (293 K and 4.2 K) and for $293 \mathrm{~K}$ (PMMA and $\mathrm{SiO}_{2}$ ). The vertical dashed line in (d) marks the region of diffusivities which are dominated by the experimental noise (values below $10 \mathrm{meV}^{2} \mathrm{~s}^{-1}$ ). The horizontal lines represent maximum and minimum values of the ratio of complementary functions for PMMA and $\mathrm{SiO}_{2}$ at $293 \mathrm{~K}$. 
4.2 $\mathrm{K}$ for $\mathrm{SiO}_{2}$ and PMMA. The apparently "multicomponent" behaviour indicates, in coherence with our expectations, that the spectral diffusion cannot be associated with a simple Brownian-type diffusion of a single distinct emissive state, but with the presence of at least two emissive states of discernible energy. This is in line with the assumption that spectral diffusion takes place e.g. as a result of conformational changes where different conformers are separated by energy barriers and exhibit different realisation probabilities. The cumulative distributions can be fitted by a sum of $i$ error functions (see eqn (3)), where each value of $D_{\mathrm{s}, i}$ describes a well defined "diffusion" process. ${ }^{40}$ The individual fitting function is described by

$$
\begin{gathered}
C_{\mathrm{s}}\left(d_{\mathrm{s}}\right)=\sum_{i} A_{i}\left[1-\operatorname{erf}\left(\sqrt{\frac{d_{\mathrm{s}}}{2 D_{\mathrm{s}, i}}}\right)\right] \\
\sum_{i} A_{i}=1 .
\end{gathered}
$$

$A$ is the amplitude of the fitting function which is assigned to one diffusion process. The error function in general is defined as

$$
\operatorname{erf}(z)=\frac{2}{\sqrt{\pi}} \int_{0}^{z} \mathrm{e}^{-t^{2}} \mathrm{~d} t
$$

Experiments at $293 \mathrm{~K}$ for DPP in PMMA show a broad distribution of "spectral diffusion coefficients". Thereby the amplitudes for large spectral jumps are low indicating their infrequence. The curves in Fig. 7(a) are fitted by a sum of 3 error functions where the first one represents noise related values, the second one small spectral diffusion processes and the third one large spectral diffusion processes. At $4.2 \mathrm{~K}$ the overall behaviour remains very similar but the probability for large and small spectral jumps is reduced by at least one order of magnitude as can be seen from the ratio of the two curves for PMMA at $293 \mathrm{~K}$ and $4.2 \mathrm{~K}$ shown in Fig. 7(c). As expected, lowering the temperature freezes the spectral diffusion very effectively for large and small spectral jumps, which have to be assigned to conformational changes and polymer dynamics that are not completely frozen out as can be seen from Fig. 3.

Room temperature data for DPP on $\mathrm{SiO}_{2}$ show a somewhat different behaviour. For diffusivities $d_{\mathrm{s}}<200 \mathrm{meV}^{2} \mathrm{~s}^{-1}$ there seems to be also a similar distribution of various diffusion processes but with larger diffusion coefficients than for DPP in PMMA. However, for $d_{\mathrm{s}}>200 \mathrm{meV}^{2} \mathrm{~s}^{-1}$ this behaviour crosses over to an apparent single diffusion coefficient of $D_{\mathrm{s}}\left(\mathrm{SiO}_{2}, 293 \mathrm{~K}\right)=(659 \pm 24) \mathrm{meV}^{2} \mathrm{~s}^{-1}$ (gained from the fitting function in Fig. 7(a)), which we assign to be related to conformational changes. Lowering the temperature nearly freezes large conformational jumps (see Fig. 7(b)) and the overall behaviour at $4.2 \mathrm{~K}$ is very similar to that in PMMA. The most obvious difference between the two environments occurs for small spectral jumps. In the case of $\mathrm{SiO}_{2}$ the reduction of such fluctuations is much less dependent on the temperature. Whereas in the polymer matrix the temperature plays a more important role. This is reflected in the much higher probability for small fluctuations at $293 \mathrm{~K}$ in PMMA (Fig. 7(d), which shows the ratio of the complementary distribution function for PMMA and for $\mathrm{SiO}_{2}$ at $293 \mathrm{~K}$ ). It is clearly visible that the amount of small spectral fluctuations overweighs in comparison to $\mathrm{SiO}_{2}$. In contrast large spectral changes in PMMA in the region of $500 \mathrm{meV}^{2} \mathrm{~s}^{-1}$ appear with a probability of only $20 \%$ compared to $\mathrm{SiO}_{2}$. The described temperature and matrix dependence can also be identified in the spectral jump width distribution shown in Fig. 5 and 6.

The distribution plot of spectral jumps for PP and DAP in PMMA provides strong evidence for the existence of individual two-level systems (Fig. 6), which are obviously not seen in Fig. 7. However, from Fig. 6 it becomes clear that such an "isolated" two-level system is rare and not detectable in the cumulative distribution shown in Fig. 7 for all molecules, but should be seen in the cumulative distribution of a single molecule. The spectral time trace and the cumulative distribution are shown in Fig. 8 for a single PP molecule. Contrary to results shown in Fig. 7 an upper limit of diffusivity becomes visible in the diffusivity range around $500 \mathrm{meV}^{2} \mathrm{~s}^{-1}$.

Fig. 8(a) clearly shows that two distinct levels can be assigned with a separation of about $15 \mathrm{meV}$ to $25 \mathrm{meV}$. This separation is very close to the "side bands" of the jump width distribution in Fig. 6 (middle). We assign this behaviour to a coupling of the optical transition to a distinct and well defined two-level system repeatedly reported in the literature. ${ }^{7,8}$ Such distinct transitions within the two-level system are observed more often for PP and DAP in PMMA as compared to those for DPP on $\mathrm{SiO}_{2}$ and in PMMA, though they are also present in these systems. We like to mention that the assigned two-level systems are not necessarily related to TLS of the amorphous materials due to the fact that the related energy differences between the two states appear also on $\mathrm{SiO}_{2}$ but with a lower probability. This is additionally confirmed by recent publications on the influence of TLS (contributing only a

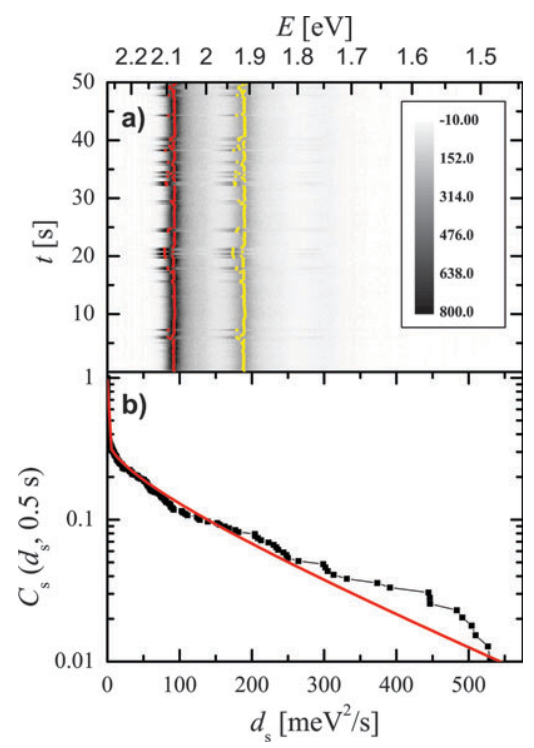

Fig. 8 (a) Spectral time trace of a single PP molecule in PMMA at $4.2 \mathrm{~K}$. The time evolution exhibits discrete jumps between two states. (b) Complementary function for the spectral time trace shown above. The red line represents a fit consisting of two error functions. The fitting procedure reveals a spectral diffusion coefficient of $D_{\mathrm{s}}=(110 \pm 2) \mathrm{meV}^{2} \mathrm{~s}^{-1}$. 

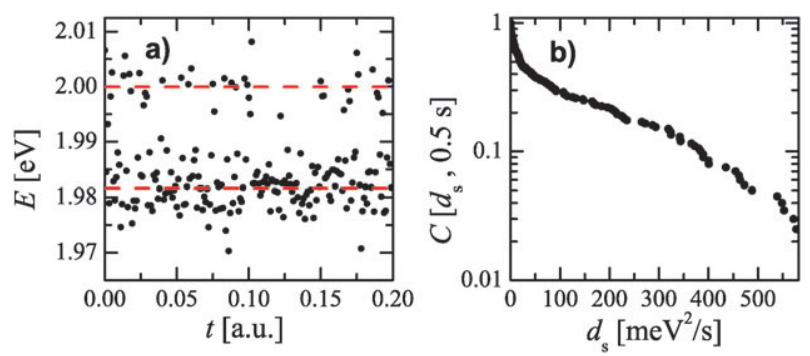

Fig. 9 (a) Simulated time trace of fluorescence emission peaks with energies of the two states of 2.000 and $1.982 \mathrm{eV}$ (indicated by the red dashed lines) and a Gaussian distributed noise of $3 \mathrm{meV}$. The ratio of occupation of the energetically high and low levels was chosen to be the same as that experimentally observed (1:5.5, see Fig. 8(a)). (b) Complementary cumulative distribution function representing the two-level systems time trace in (a).

fraction of $1 \mathrm{meV}$ to energy differences) and low frequency modes (causing predominantly broadenings of the line width) on line shapes and spectral fluctuations. ${ }^{7}$ From this we conclude that the polymer dynamics allow for distinct switching between specific intramolecular conformers.

A cumulative distribution has been simulated for jumps in a two-level system while considering experimental noise. The energy difference between the two states and the ratio of occupation of energetically lower and higher states were estimated from the experimental data of Fig. 8(a) to $\Delta E=18 \mathrm{meV}$ and $r_{\mathrm{TLS}}=5.5$. In the simulation, we assumed a noise of $3 \mathrm{meV}$ for the position of the maximum of the spectrum, with a Gaussian distribution around the two energy levels. It has to be emphasised that this is only an approximation. For a more accurate description of the noise one has to consider that a mixture of the two states can appear due to the integration time of $0.5 \mathrm{~s}$. This will cause intermediate states within the spectral time trace. The results for simple Gaussian distributed noise around the states are shown in Fig. 9. Comparison with experiment shows a qualitative agreement on a single molecule level. Both, experimental and simulated curves exhibit two components: one fast decaying component which represents the noise and a second slow decaying component which can be assigned to the observed diffusion processes. This confirms the assumption that the low temperature spectral fluctuations for PP in PMMA can be described via a two-level system.

\section{Conclusions}

The outlined single molecule spectroscopy studies of perylene diimide molecules under different conditions revealed a complex behaviour of spectral line widths and spectral diffusion processes.

The spectral line widths did not show the expected strong temperature dependence known from low temperature fluorescence excitation experiments. Even at $4.2 \mathrm{~K}$ emission line widths were only reduced by a factor of about 2 . We assign this unexpected broadening behaviour to spectral fluctuations on a time scale far below our experimental time resolution. Less than $5 \%$ of the investigated molecules exhibit narrow emission lines in the region of our spectral resolution confirming that line broadening can in principle be reduced by lowering the temperature. The temperature influence on observable spectral dynamics in the time scale of $0.5 \mathrm{~s}$ showed, in contrast, a much stronger correlation. Large spectral jumps of up to $120 \mathrm{meV}$ could be especially observed on $\mathrm{SiO}_{2}$ at room temperature due to the high degree of freedom of the bay groups. As this is not the case in PMMA large spectral fluctuations were already reduced at $293 \mathrm{~K}$. At low temperatures these phenomena were almost completely reduced for DPP molecules. In contrast to these observations a slight difference in the chemical structure of the dye molecules (PP and DAP) changed the situation in PMMA at low temperatures dramatically. For PP and especially DAP a strong enhancement of spectral fluctuations was observed at $4.2 \mathrm{~K}$ which we interpret as a coupling of the butyl chain(s) to the surrounding environment.

\section{Acknowledgements}

We like to thank Frank Würthner (Würzburg University) for a kind donation of DPP, PP and DAP. We acknowledge financial support by the German Science Foundation within the research unit "From Local Constraints to Macroscopic Transport" (SFG 877) and the DAAD for supporting exchange between the University of Chemnitz and the University of Buenos Aires. PFA is a Research Fellow from CONICET (Consejo Nacional de Investigaciones Científicas y Técnicas, Argentina).

\section{Notes and references}

1 F. Kulzer, T. Xia and M. Orrit, Angew. Chem., Int. Ed., 2010, 40, 854-866.

2 C. Tietz, R. Daum, A. Dräbenstedt, J. Schuster, L. Fleury, A. Gruber, J. Wrachtrup and C. von Borczyskowski, Chem. Phys. Lett., 1998, 282, 164-170.

3 J. Schuster, F. Cichos, J. Wrachtrup and C. von Borczyskowski, Single Mol., 2000, 1, 299.

4 Molecular Physics, 2009, 107, Number 18, Special Issue on Spectral Dynamics and Related Topics, ISSN: 0026-8976.

5 D. Wöll, E. Braeken, A. Deres, F. C. De Schryver, H. Uji-i and J. Hofkens, Chem. Soc. Rev., 2009, 38, 313.

6 R. A. L. Vallee, W. Paul and K. Binder, J. Chem. Phys., 2010, 132, 034901.

7 Y. Vainer, A. Naumov, M. Bauer and L. Kador, Phys. Rev. Lett., 2006, 97, 185501-1-185501-4.

8 A. Boiron, P. Tamarat, B. Lounis, R. Brown and M. Orrit, Chem. Phys., 1999, 247, 119-132.

9 A. V. Naumov, Y. G. Vainer, M. Bauer and L. Kador, J. Chem. Phys., 2002, 116, 8132.

10 A. V. Naumov, Y. G. Vainer, M. Bauer and L. Kador, J. Chem. Phys., 2003, 119, 6296.

11 R. A. L. Vallée, M. Cotlet, M. Van Der Auweraer, J. Hofkens, K. Müllen and F. C. De Schryver, J. Am. Chem. Soc., 2004, 126, 2296-2297.

12 J. Baier, M. Richter, R. Cogdell, S. Oellerich and J. Köhler, Phys. Rev. Lett., 2008, 100, 018108.

13 M. Brecht, H. Studier, V. Radics, J. B. Nieder and R. Bittl, J. Am. Chem. Soc., 2008, 130, 17487-17493.

14 E. Lang, F. Würthner and J. Köhler, ChemPhysChem, 2005, 6, 935-941.

15 J. Baier, M. Richter, R. Cogdell, S. Oellerich and J. Köhler, Phys. Rev. Lett., 2008, 100, 018108.

16 C. J. Wei, C. Lu, Y. H. Kim and D. A. Bout, J. Fluoresc., 2007, 17, 797-804. 
17 H. Uji-i, S. M. Melnikov, A. Deres, G. Bergamini, F. De Schryver, A. Herrmann, K. Müllen, J. Enderlein and J. Hofkens, Polymer, 2006, 47, 2511-2518.

18 R. A. L. Vallee, T. Rohand, N. Boens, W. Dehaen, G. Hinze and T. Basche, J. Chem. Phys., 2008, 128, 154515.

19 R. A. L. Vallée, M. Van Der Auweraer, F. C. De Schryver, D. Beljonne and M. Orrit, ChemPhysChem, 2005, 6, 81-91.

20 R. Vallée, M. Van der Auweraer, W. Paul and K. Binder, Phys. Rev. Lett., 2006, 97, 217801-1-217801-4.

21 R. Vallée, N. Tomczak, L. Kuipers, G. Vancso and N. van Hulst, Phys. Rev. Lett., 2003, 91, 038301-1-038301-4.

22 R. A. L. Vallée, M. Cotlet, M. Van Der Auweraer, J. Hofkens, K. Müllen and F. C. De Schryver, J. Am. Chem. Soc., 2004, 126, 2296-2297.

23 C. Blum, F. Stracke, S. Becker, K. Müllen and A. J. Meixner, J. Phys. Chem. A, 2001, 105, 6983-6990.

24 R. A. L. Vallée, P. Marsal, E. Braeken, S. Habuchi, F. C. De Schryver, M. Van der Auweraer, D. Beljonne and J. Hofkens, J. Am. Chem. Soc., 2005, 127, 12011-12020.

25 K. L. Wustholz, E. D. Bott, B. Kahr and P. J. Reid, J. Phys. Chem. C, 2008, 112, 7877-7885.

26 F. Würthner, Pure Appl. Chem., 2006, 78, 2341-2349.

27 F. Würthner and A. Sautter, Org. Biomol. Chem., 2003, 1, 240-243.

28 J. Hofkens, T. Vosch, M. Maus, F. Köhn, M. Cotlet, T. Weil, A. Herrmann, K. Müllen and F. C. De Schryver, Chem. Phys. Lett., 2001, 333, 255-263.
29 E. Fron, G. Schweitzer, P. Osswald, F. Würthner, P. Marsal, D. Beljonne, K. Müllen, F. C. De Schryver and M. Van der Auweraer, Photochem. Photobiol. Sci., 2008, 7, 1509.

30 D. Kowerko, J. Schuster and C. von Borczyskowski, Mol. Phys., $2009,10,1$.

31 J. Hohlbein and C. G. Hubner, J. Chem. Phys., 2008, 129, 094703.

32 S. Krause, D. Kowerko, R. Börner, C. G. Hübner and C. von Borczyskowski, ChemPhysChem, DOI: 10.1002/cphc.201000678.

33 E. Lang, PhD thesis, University of Bayreuth, 2006.

34 V. P. Biju, J. Y. Ye and M. Ishikawa, J. Phys. Chem. B, 2003, 107, 10729-10735.

35 O. Mirzov, T. Pullerits, F. Cichos, C. von Borczyskowski and I. Scheblykin, Chem. Phys. Lett., 2005, 408, 317-321.

36 T. Pullerits, O. Mirzov and I. G. Scheblykin, J. Phys. Chem. B, 2005, 109, 19099-19107.

37 C. Hellriegel, J. Kirstein, C. Bräuchle, V. Latour, T. Pigot, R. Olivier, S. Lacombe, R. Brown, V. Guieu, C. Payrastre, A. Izquierdo and P. Mocho, J. Phys. Chem. B, 2004, 108, 14699-14709.

38 M. J. Saxton and K. Jacobson, Annu. Rev. Biophys. Biomol. Struct., 1997, 26, 373-399.

39 D. Täuber, J. Schuster, M. Heidernätsch, M. Bauer, G. Radons and C. von Borczyskowski, Diffus. Fundam., 2009, 11, 107.

40 C. Jung, J. Kirstein, B. Platschek, T. Bein, M. Budde, I. Frank, K. Müllen, J. Michaelis and C. Bräuchle, J. Am. Chem. Soc., 2008, 130, $1638-1648$. 